\title{
Advanced Methods of 3D Stress Intensity Factor Assessment
}

\author{
Jinhua TAN ${ }^{1,}$, , Rui Zhang ${ }^{1, b}$ Xiangping Chen ${ }^{1, c}$ \\ ${ }^{1}$ School of transportation, Wuhan University of Technology, Wuhan, P.R. China \\ E-mail: ${ }^{a}$ tan-jh@163.com, ${ }^{b} 1539211678 @ q q . c o m,{ }^{c}$ chxp91021@sohu.com
}

Keywords: Stress intensity factor; Interaction Integrals; J-Integral evaluation, Energy-Release Rate

\begin{abstract}
This paper explains and demonstrates how to get stress intensity factors using different evaluation methods for a 3D plate with a straight through-thickness crack. The methods include interaction integrals, J-integral evaluation and energy-release rate. All results from these methods are compared with others, and some useful information about the accuracy and the applicability of these methods obtained.
\end{abstract}

\section{Introduction}

Structural design concepts traditionally use a strength-of-material approach for designing a structure. This method does not consider the elevated stress levels due to the cracks. The presence of such stresses can lead to catastrophic failure of the structure.

Fracture mechanics accounts for the cracks or flaws in a structure. The fracture mechanics method to design of structures includes crack size as one important variable, which can anticipate the adverse elevated stress levels and avoid the unexpected collapse accident.

Fracture analysis is typically carried out either using the energy criterion or the stress-intensity-factor criterion. The advanced approaches include interaction integrals, J-integral evaluation too. All these method are used to get stress intensity factors for a 3D plate with a straight through-thickness crack in this paper, and the results are compared with others, and some useful information about the accuracy and the applicability of these methods provided.

\section{Evaluation approaches of stress intensity factors(SIFs)}

General Theory. For a linear elastic material the stress and strain fields ahead of the crack tip are expressed as:

$$
\begin{aligned}
& \sigma_{i j}=-\frac{K}{\sqrt{r}} f_{i j}(\theta) \\
& \varepsilon_{i j}=-\frac{K}{\sqrt{r}} g_{i j}(\theta)
\end{aligned}
$$

where $\mathrm{K}$ is the stress-intensity factor, $\mathrm{r}$ and $\theta$ are coordinates of a polar coordinate system. These equations apply to any of the three fracture modes.

For a Mode I crack, the stress field is given as:

$$
\begin{aligned}
& \sigma_{\mathrm{x}}=\frac{\mathrm{K}_{1}}{\sqrt{2 \pi \mathrm{r}}} \cos \left(\frac{\theta}{2}\right)\left(1-\sin \left(\frac{\theta}{2}\right) \sin \left(\frac{3 \theta}{2}\right)\right) \\
& \sigma_{\mathrm{y}}=\frac{\mathrm{K}_{1}}{\sqrt{2 \pi \mathrm{r}}} \cos \left(\frac{\theta}{2}\right)\left(1+\sin \left(\frac{\theta}{2}\right) \sin \left(\frac{3 \theta}{2}\right)\right) \\
& \sigma_{\mathrm{xy}}=\frac{\mathrm{K}_{1}}{\sqrt{2 \pi \mathrm{r}}} \cos \left(\frac{\theta}{2}\right) \sin \left(\frac{\theta}{2}\right) \cos \left(\frac{3 \theta}{2}\right)
\end{aligned}
$$

For center cracked plate geometry, the stress intensity factor for this problem was calculated by Isida as equal to:

$$
K_{\mathrm{I}}=1.33+\sigma \sqrt{\pi a}
$$

Energy-Release Rate.The energy-release rate $G$ is defined in elastic materials as the rate of change of potential energy released from a structure when a crack opens. 
The energy-release rate is given by:

$$
G=\frac{\pi \sigma^{2} a}{E}
$$

At the moment of fracture, $G$ is equal to the critical energy-release rate $G c$, a function of the fracture toughness. The value of $\mathrm{Gc}$ for a material can be determined via a relatively straightforward set of crack experiments.For a single-fracture mode, the stress-intensity factor and the energy-release rate are related by: $G=\frac{K^{2}}{E^{c}}$,

where $\mathrm{G}$ is the energy-release rate, $E^{\prime}=\frac{E}{1-v^{2}}$ for plane strain, and $E^{\prime}=E$ for plane stress. (E is the material Young's modulus, and $v$ is the Poisson's ratio.)

J-Integral. J-Integral is one of the most widely accepted parameters for elastic-plastic fracture mechanics, and it can be looked as a Stress-Intensity Factor too. Hutchinson and Rice and Rosengren independently showed that the J-Integral characterizes the crack-tip field in a nonlinear elastic material. They each assumed a power law relationship between plastic strain and stress.

The J-Integral evaluation is based on the domain integral method by Shih. The domain integration formulation applies area integration for 2-D problems and volume integration for 3-D problems. The J-Integral is defined as follows :

$$
J=\operatorname{Lim}_{\Gamma \rightarrow 0} \int_{\Gamma_{0}}\left[(w+T) \delta_{l i}-\sigma_{i j} \frac{\partial u_{j}}{\partial x_{l}}\right] n_{i} d \Gamma
$$

where $\mathrm{W}$ is the strain energy density, $\mathrm{T}$ is the kinematic energy density, $\sigma$ represents the stresses, $\mathrm{u}$ is the displacement vector, and $\Gamma$ is the contour over which the integration is carried out.

For a 2-D problem, body forces within the integration of area, and pressure on the crack surface, the domain integral representation of the J-Integral is given by:

$$
J=\int_{A}\left[\sigma_{i j} \frac{\partial u_{j}}{\partial x_{1}}-w_{1 i}\right] \frac{\partial q}{\partial x_{i}} d A
$$

Where

$$
\begin{gathered}
\omega=\omega^{e}+\omega^{p}=\int_{0}^{\varepsilon_{k l}^{e}} \sigma_{i j} d \varepsilon_{i j}^{e}+\int_{0}^{\varepsilon_{k l}^{p}} S_{i j} d \varepsilon_{i j}^{p} \\
S_{i j}=\sigma_{i j}-\frac{1}{3} \sigma_{k k} \delta_{i j}
\end{gathered}
$$

where $\sigma_{i j}$ is the stress tensor, $u_{j}$ is the displacement vector, $w$ is the strain energy density, $\delta_{i j}$ is the Kronecker delta, $x_{i}$ is the coordinate axis, and $q$ is referred to as the crack-extension vector.

$$
\begin{gathered}
q\left(X_{i}\right)=\sum_{I=1}^{m} N_{I} q_{I} \\
\frac{\partial q\left(X_{i}\right)}{\partial X_{i}}=\sum_{I=1}^{m} \sum_{p=1}^{20 r 3} \frac{\partial N_{I}}{\partial \eta_{p}} \frac{\partial \eta_{p}}{\partial X_{j}} q_{I}
\end{gathered}
$$

Equation (6) can be the following equation when Gaussian integral method used:

$$
J=\sum_{A} \sum_{p=1}^{p}\left\{\left[\left(\sigma_{i j} \frac{\partial U_{j}}{\partial X_{1}}-\omega \delta_{1 i}\right) \frac{\partial q}{\partial X_{i}}\right]|J|\right\}_{p} W_{p}
$$

Wherein: $P$ is integration points of a element, $|\mathbf{J}|$ is the Jacobian and $w_{p}$ is the weight of Gaussian integration points.

For the 3-D problem, domain integral representation of the J-Integral becomes a volume integration, which again is evaluated over a group of elements, and the principal is similar to the 2-D problem. And there is equation(10) for J integration: 


$$
\bar{J} \Delta L=\int_{V}\left\{\left[\sigma_{i j} \frac{\partial U_{j}}{\partial X_{1}}-\omega \delta_{1 i}\right] \frac{\partial q}{\partial X_{i}}\right\} d V
$$

Interaction Integrals. Similar to the domain integral method for J-Integral evaluation, the interaction integral method for stress-intensity factors calculation applies area integration for 2-D problems and volume integration for 3-D problems. The interaction integral is defined as

$$
J=-\int_{V} q_{i, j}\left(\sigma_{k i} \varepsilon_{k i}^{a u x} \delta_{i j}-\sigma_{k j}^{a u x} u_{k, i}-\sigma_{k j} u_{k, i}^{a u x}\right) d V / \int_{S} \delta q_{n} d S
$$

where $\sigma_{i j}, \varepsilon_{i j}, u_{i}$ are the stress, strain and displacement respectively, $\sigma_{i j}^{a u x}, \varepsilon_{i j}^{a u x}, u_{i}^{a u x}$ are the stress, strain and displacement of the auxiliary field, and $q_{\mathrm{i}}$ is the crack-extension vector.

The interaction integral is associated with the stress-intensity factors as

$$
\mathrm{I}=\frac{2}{\mathrm{E}^{*}}\left(\mathrm{~K}_{1} \mathrm{~K}_{1}^{\mathrm{aux}}+\mathrm{K}_{2} \mathrm{~K}_{2}^{\mathrm{aux}}\right)+\frac{1}{\mu} \mathrm{K}_{3} \mathrm{~K}_{3}^{\mathrm{aux}}
$$

where $K_{i}(i=1,2,3)$ are the mode I, II and III stress-intensity factors, ${ }_{i}^{a u x}(i=1,2,3)$ are the auxiliary mode I, II and III stress-intensity factors, $E^{*}=$ E for plane stress and $E^{*}=E /\left(1-\square v^{2}\right)$ for plane strain, $\mathrm{E}$ is the Young's modulus, $v$ is the Poisson ratio, and $\mu$ is the shear modulus.

\section{Modelling and calculation of SIFs}

Modelling of a center cracked plate. For center cracked plate geometry is shown in Fig. 1. Given the symmetry, only a quarter of the panel was modeled by a structure analysis software, and got the SIFs from the different approaches through the post process.The main stress S1 is shown in Fig.2.

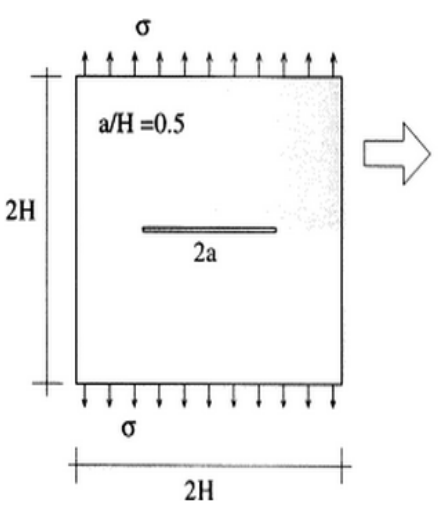

Fig.1 Center cracked plate

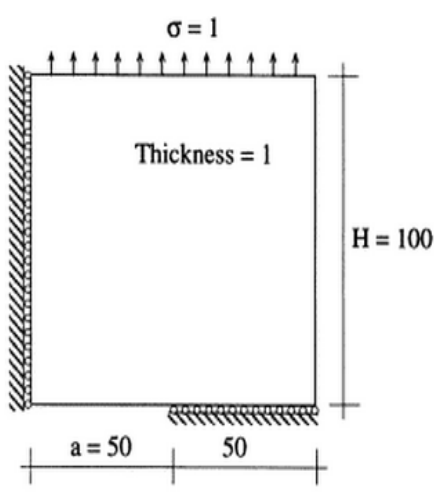

The results of SIFs. All results in 10 contours are listed as follows, and the SIFs obtained too.

\section{J-integral \\ ***** POST1 J-INTEGRAL RESULT LISTING ***** \\ CrackID $=2$ \\ Crack Front Node $=46$ \\ Contour Values $=-398.61 \quad 11450 . \quad 12489 . \quad 12572$. \\ Contour Values $=11295 . \quad 11187 . \quad 11124 . \quad 11093$. \\ Contour Values $=11081 . \quad 11079$.}

The maximum 11876 is the J-integral value.

In the case of plane stress state, the K2 can be obtained by: 


$$
\mathrm{K}=\sqrt{E J}=\sqrt{2.1 \mathrm{e} 11 * 12575}=4.994 \mathrm{E} 7
$$

In the case of plane stress state, the K2 can be obtained by:

\section{Interaction integral}

$$
K=\sqrt{\frac{E}{1-v^{2}}}=5.235 \mathrm{E} 7
$$

Results of $\mathrm{K}$ in 10 contours via interaction integral method:

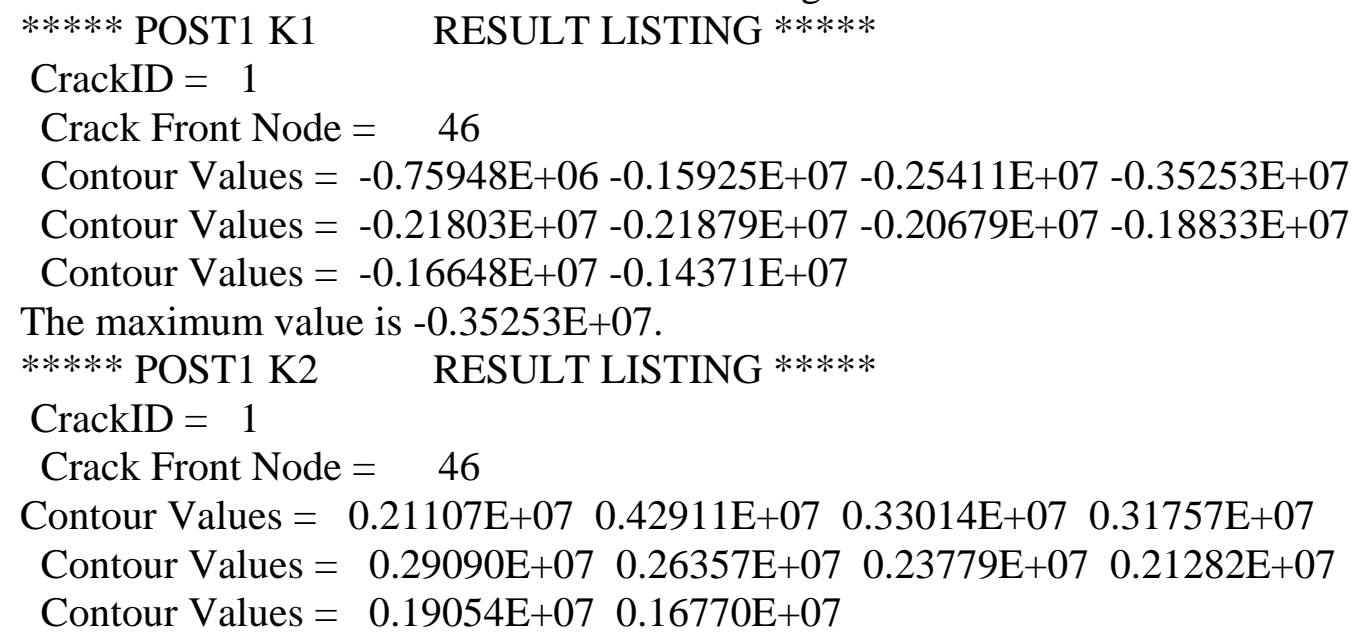

\section{Conclusions}

Area and volume integrals offer much better accuracy than contour integral and surface integrals, and are much easier to implement numerically. In comparison to the traditional method, the interaction integral method offers better accuracy, fewer mesh requirements, and ease to use.

\section{References}

[1] Gerd Heber, Paul A. Wawrzynek etc., Three-dimensional, parallel, finite element simulation of fatigue crack growth in a spiral bevel pinion gear, Engineering Fracture Mechanics, Volume 72, Issue 8, May 2005, Pages 1148-1170

[2] C. A. Duarte,O.N. Hamzeh, T.J. Liszka, W.W. Tworzydlo. A generalized finite element method for the simulation of three-dimensional dynamic crack propagation [J]. Compu. Methods Appli. Mech. Engrg. 2001,190:2227-2262.

[3] Roychowdhury S, Dodds RH Jr. Effect of T-stress on fatigue crack closure in 3D small-scale yielding. International Journal of Solids and Structures, 2004, 41: 2581 2606.

[4] Zhang Bin, Guo W, She CM at al. Tz constraints around quarter-elliptical corner cracks in elastic plates subjected to uniform tension loading. International Journal of Fatigue, 2005 (in review, NA483).

[5] Elber W. Fatigue crack propagation. PhD Thesis, University New South Wales, Australia; 1968

[6] Paris, P. C. and Erdogan, F. (1963) . A critical analysis of crack propagation laws. Journal of Basic Engineering, ASCE, 85, 528-534. 